\title{
CINÉTICA DE CRECIMIENTO DE LAS FASES NITRURADAS EN HIERRO PURO ARMCO OBTENIDAS POR EL PROCESO DE NITRURACIÓN EN POLVO
}

\author{
M. Elías-Espinosa ${ }^{[a]}$, Y. Muñoz-Sánchez ${ }^{[b]}$, A. Cruz-Avilés ${ }^{[b]}$, M. Ortiz-Domínguez ${ }^{[b]}$, J. A. Cid Blancas ${ }^{[c]}$ \\ ${ }^{\text {[a] }}$ Profesor-Investigador del Instituto Tecnológico y de Estudios Superiores de Monterrey-ITESM Campus Santa Fe \\ ${ }^{[b]}$ Profesor-Investigador en la Escuela Superior de Ciudad Sahagún de la Universidad Autónoma del Estado de Hidalgo, Hidalgo, \\ México \\ ${ }^{[c]}$ Estudiante de Ingeniería Mecánica en la Escuela Superior de Ciudad Sahagún de la Universidad Autónoma del Estado de Hidalgo, \\ Hidalgo, México
}

\section{Resumen}

El presente trabajo estudia el crecimiento de las capas de nitruros de hierro generadas por el proceso de nitruración en polvo, sobre la superficie de un hierro puro Armco. El tratamiento termoquímico de nitruración en polvo consiste en un polvo rico en nitrógeno $\left(\mathrm{CaCN}_{2}\right)$, con el que se cubren las piezas que se desean endurecer empleando la técnica de empaquetamiento en caja. Las variables utilizadas en este estudio fueron: cuatro valores de temperatura $(773,798,823$ y 848 K) con cinco tiempos de tratamiento $(2,4,6,8$ y $12 \mathrm{~h})$ y tres relaciones activador/polvo rico en nitrógeno $(0.20,0.25$ y 0.35$)$. Empleando las ecuaciones de balance de masa en las interfaces de crecimiento $\varepsilon-\mathrm{Fe}_{2} \mathrm{~N}_{1-\gamma} / \gamma^{\prime}-\mathrm{Fe}_{4} \mathrm{~N}_{1-x} \mathrm{y} \gamma^{\prime}-\mathrm{Fe}_{4} \mathrm{~N}_{1-\mathrm{x}} / \mathrm{Fe}$, se determinó la movilidad del boro en la superficie del substrato $\left(D_{\mathcal{E}-F e_{2} N_{1-y}}\right.$ y $\left.D_{\gamma^{\prime}-F e_{4} N_{1-x}}\right)$, considerando la influencia de los tiempos de incubación para cada fase de crecimiento $\left(t_{0}^{\varepsilon-F e_{2} N_{1-y}}\right.$ y $\left.t_{0}^{\gamma^{\prime}-F e_{4} N_{1-x}}\right)$. Para validar el modelo de difusión propuesto, el valor simulado para los espesores de las capas $\varepsilon-\mathrm{Fe}_{2} \mathrm{~N}_{1-y} \mathrm{y} \gamma^{\prime}-\mathrm{Fe}_{4} \mathrm{~N}_{1-\mathrm{x}}$ se comparó con un valor experimental respectivamente. La morfología de las capa fue observada a través de Microscopía Electrónica de Barrido (SEM) y Microscopía Óptica. Las fases $\varepsilon-\mathrm{Fe}_{2} \mathrm{~N}_{1-y}$ y $\gamma^{\prime}-\mathrm{Fe}_{4} \mathrm{~N}_{1-\mathrm{x}}$ fueron identificadas por el método de difracción de rayos $X$.

Palabras Clave: Nitruración, Modelo de Difusión, Energía de activación, Tiempo de incubación, Tratamiento Termoquímico.

\section{Abstract}

The present work studies the growth of iron nitride layer produced by powder pack nitriding process on the surface of a pure iron Armco. The powder pack nitriding process uses nitrogen rich powder ( $\mathrm{CaCN} 2)$. The variables used in this study were four values of temperature $(773,798,823$ 
and $848 \mathrm{~K})$ with five treatment times $(2,4,6,8$ and $12 \mathrm{~h})$ and three activator/powder ratios $(0.20$, 0.25 and 0.35 ) were used for the set of experimental conditions of the powder-pack nitriding process. Using the mass balance equations at interfaces growth $\varepsilon-\mathrm{Fe}_{2} \mathrm{~N}_{1-y} / \gamma^{\prime}-\mathrm{Fe}_{4} \mathrm{~N}_{1-x}$ y $\gamma^{\prime}-\mathrm{Fe}_{4} \mathrm{~N}_{1-x} / \mathrm{Fe}$, mobility of boron it was determined on the surface of the substrate $\left(D_{\varepsilon-F e_{2} N_{1-y}}\right.$ and $\left.D_{\gamma^{\prime}-F e_{4} N_{1-x}}\right)$, considering the influence of the incubation times for each phase of growth $\left(t_{0}^{\varepsilon-F e_{2} N_{1-y}} \mathrm{y}\right.$ $\left.t_{0}^{\gamma^{\prime}-F e_{4} N_{1-x}}\right)$. To validate the proposed diffusion model, the simulated value for the thickness of the $\varepsilon-\mathrm{Fe}_{2} \mathrm{~N}_{1-y}$ y layers $\gamma^{\prime}-\mathrm{Fe}_{4} \mathrm{~N}_{1-x}$ was compared with an experimental value respectively. The morphology of the layer was observed by Scanning Electron Microscopy (SEM) and Optical Microscopy. The $\varepsilon-\mathrm{Fe}_{2} \mathrm{~N}_{1-y}$ and $\gamma^{\prime}-\mathrm{Fe}_{4} \mathrm{~N}_{1-x}$ phases were identified by $x$-ray diffraction method.

Keywords: Nitriding, Diffusion Model, Activation energy, Incubation time, Thermochemical treatment.

\section{Introducción}

La nitruración es un tratamiento termoquímico mediante el cual se consigue que se difunda nitrógeno atómico sobre la superficie de un substrato, esto genera una monocapa $\gamma^{\prime}-\mathrm{Fe}_{4} \mathrm{~N}_{1-\mathrm{x}} \mathrm{O}$ una bicapa $\varepsilon-\mathrm{Fe}_{2} \mathrm{~N}_{1-\mathrm{y}} / \gamma^{\prime}-\mathrm{Fe}_{4} \mathrm{~N}_{1-\mathrm{x}}$. Estas capas mejoran las propiedades superficiales de los sustratos: aumento de dureza, resistencia al desgaste, resistencia a la fatiga, alta resistencia a la cavitación, y buena resistencia a la corrosión [1-3].

La nitruración en polvo es un tratamiento termoquímico que utiliza el proceso de empaquetamiento y es adecuado para componentes ingenieriles con geometrías complejas y con distintos requisitos para la formación de capas nitruradas. La ventaja de este método es su bajo costo en comparación con otras tecnologías, tales como nitruración iónica o nitruración postdescarga microondas [2,3]. La nitruración en polvo utiliza una mezcla de Cianamida de Calcio $\left(\mathrm{CaCN}_{2}\right)(23.5 \% \mathrm{~N})$, este compuesto produce nitrógeno cuando se disocia a temperaturas superiores a los $673 \mathrm{~K}$, como se muestra en la ecuación (1) [4-6].

$$
2 \mathrm{CaCN}_{2}+3 \mathrm{O}_{2} \rightarrow 2 \mathrm{CaCN}_{2}+2 \mathrm{~N}_{2} .
$$

\subsection{MODELO DE DIFUSIÓN}

Se propone un modelo matemático para determinar la cinética de crecimiento en la monocapa $\gamma^{\prime}$ $\mathrm{Fe}_{4} \mathrm{~N}_{1-x}$ y/o bicapa $\varepsilon-\mathrm{Fe}_{2} \mathrm{~N}_{1-y} / \gamma^{\prime}-\mathrm{Fe}_{4} \mathrm{~N}_{1-x}$ en la superficie del hierro Armco empleando el proceso de nitruración en polvo. El modelo se plantea a partir del estado estacionario que describe el proceso de difusión, el movimiento de las fases, las caídas de concentración y el balance de masa en las interfases de crecimiento. Además, se parte de las suposiciones que las fases no comienzan a formarse instantáneamente $\left(t^{\prime}=0\right)$ sino a partir de un cierto tiempo de incubación $t_{0}^{\left(\mathrm{Fe}_{2} \mathrm{~N}_{1-y}, \mathrm{Fe}_{4} \mathrm{~N}_{1-x}\right)}(T)$, en el cual se representa la formación de una película base, la cual termina con la aparición de los primeros nitruros de hierro. El modelo asume un perfil de concentración 
lineal de nitrógeno para cada una de las fases formadas ( $\varepsilon-\mathrm{Fe}_{2} \mathrm{~N}_{1-\mathrm{y}} \mathrm{y} / \mathrm{o} \gamma^{\prime}-\mathrm{Fe}_{4} \mathrm{~N}_{1-\mathrm{x}}$ ) en la superficie del hierro Armco, como se puede observar en la Figura 1. Además el modelo propone en la superficie del substrato una composición inicial $\boldsymbol{C}_{\mathbf{0}}$. Como en la superficie del substrato es donde se presenta la adsorción de átomos de nitrógeno y estos avanzan en dirección al núcleo del substrato, se considera que existe un flujo de átomos en las interfases ( $\gamma^{\prime} /$ Fe y $\left.\varepsilon / \gamma^{\prime}\right)$ de las fases formadas. Existen modelos de difusión de nitrógeno, en donde se observa el término $\boldsymbol{C}_{a d s}^{N}$ que representa la concentración de nitrógeno absorbido en la superficie del material.

"[Aquí va la Figura 1]"

Figura 1. Perfiles de concentración del nitrógeno en las capas $\gamma^{\prime}-\mathrm{Fe}_{4} \mathrm{~N}_{1-\mathrm{x}} \mathrm{y} \varepsilon-\mathrm{Fe}_{2} \mathrm{~N}_{1-\mathrm{y}}{ }^{[9]}$.

\subsection{MODELO DE DIFUSIÓN DE LA CAPA CONSIDERANDO UNICAMENTE $\gamma^{\prime}-\mathrm{Fe}_{4} \mathrm{~N}_{1-\mathrm{x}}$}

En el modelo de difusión se considera que la fase $\gamma^{\prime}-\mathrm{Fe}_{4} \mathrm{~N}_{1-x}$ es la primera en formarse, los tiempos de incubación de las fases son independientes y diferentes $t_{0}^{F e_{2} N_{1-y}}(T)>t_{0}^{F e_{4} N_{1-x}}(T)$, así el coeficiente de difusión del nitrógeno en la fase $\gamma^{\prime}-\mathrm{Fe}_{4} \mathrm{~N}_{1-x}$, se puede determinar de manera independiente. Las condiciones iniciales y las de frontera para $C_{F e_{4} N_{1-x}}[x(t)]$ son: $t^{\prime}=0, x>0$, $C_{F_{4} N_{1-x}}[x(t)]=C_{0}, C_{F_{4} N_{1-x}}\left[x=v_{0} \approx 0\right]=C_{u p}^{\gamma^{\prime}}$ y $C_{F_{4} N_{1-x}}[x=v]=C_{\text {low }}^{\gamma^{\prime}}[7,8], v_{0}$ representa la película base de la etapa de nucleación $\approx 0$ en comparación a el espesor de $v$. Bajo la condiciones de frontera se obtiene el perfil de concentración lineal del nitrógeno en la fase $\mathrm{Fe}_{4} \mathrm{~N}_{1-\mathrm{x}}$ :

$$
C_{\mathrm{Fe}_{4} N_{1-x}}[x(t)]=C_{u p}^{\gamma^{\prime}}+\frac{C_{l o w}^{\gamma}-C_{u p}^{\gamma}}{v} x
$$

Bajo las suposiciones antes mencionadas, se puede implementar una ecuación basada en la conservación de la materia en la interfase de crecimiento:

$$
C_{\text {low }}^{\gamma^{\prime}}+C_{0}+\left(\frac{C_{\text {int }}^{\gamma^{\prime}}-C_{\text {low }}^{\gamma^{\prime}}}{2}\right)
$$

El avance de la interfase $\mathrm{Fe}_{4} \mathrm{~N}_{1-\mathrm{x}}$ y $\mathrm{Fe}$ es: $\Delta C_{N}^{\gamma^{\prime} / \mathrm{Fe}}=J_{\gamma^{\prime}} d t-J_{F e} d t$ [10] Considerando una área unitaria plana en la interface $\mathrm{Fe}_{4} \mathrm{~N}_{1-\mathrm{x}} / \mathrm{Fe}$ y una distancia $d v$ en un tiempo $d t_{v}$ ( $\boldsymbol{t}_{v}$, representa el tiempo de formación de la fase), entonces se forma un volumen $(1 \cdot 1 \cdot d v)$. Lo anterior nos indica que el incremento de $d v$ del espesor de capa de $\mathrm{Fe}_{4} \mathrm{~N}_{1-\mathrm{x}}$ en un tiempo $d t_{v}$ ocurre bajo el consumo simultáneo de la capa de espesor del $d v$ substrato. Por lo que, la acumulación de átomos de nitrógeno en el incremento $d v$ se puede expresar de la siguiente manera: 


$$
\left(\frac{C_{l o w}^{\gamma^{\prime}}-2 C_{0}+C_{u p}^{\gamma^{\prime}}}{2}\right)(1 \cdot 1 \cdot d v)=J_{\gamma^{\prime}}(x=v)\left(1 \cdot 1 \cdot d t_{v}\right)-J_{F e}(x=v+d v)\left(1 \cdot 1 \cdot d t_{v}\right)
$$

La relación entre el flujo y el gradiente de concentración de la interfase están dados por la primera ley de Fick, y como el flujo de átomos de nitrógeno es de la fase $\mathrm{Fe}_{4} \mathrm{~N}_{1-x}$ hacia la interfase $\mathrm{Fe}_{4} \mathrm{~N}_{1-x} / \mathrm{Fe}$ y de esta última hacia el sustrato $\mathrm{Fe}$, se observa que el flujo saliente se anula $\left(U_{F e}=0\right)$, debido a la baja solubilidad que tienen los átomos de nitrógeno con el substrato $\left(\boldsymbol{C}_{\mathbf{0}} \approx \mathbf{0 . 0 1 \%}\right)$ [9], por lo tanto:

La ley de crecimiento parabólico $v=k_{\gamma^{\prime}} t_{v}^{n}$, se deriva y se sustituye en la ecuación (4), por lo tanto: $v=k_{\gamma^{\prime}}\left\{t^{\prime}-\left[t_{0}^{\gamma^{\prime}}(T)\right]\right\}^{1 / 2}$, donde $\left[t_{0}^{\gamma^{\prime}}(T)\right]^{1 / 2}$ representa el tiempo de incubación. Finalmente despejando se obtiene la relación del coeficiente de difusión de la fase $\gamma^{\prime}-\mathrm{Fe}_{4} \mathrm{~N}_{1-\mathrm{x}}$ :

$$
D_{\gamma^{\prime}}=\frac{\left(C_{\text {low }}^{\gamma^{\prime}}-2 C_{0}+C_{u p}^{\gamma^{\prime}}\right)}{4\left(C_{\text {low }}^{\gamma^{\prime}}-C_{u p}^{\gamma^{\prime}}\right)}\left(k_{\gamma^{\prime}}\right) . \quad\left(m^{2} s^{-1}\right)
$$

\subsection{MODELO DE DIFUSIÓN DE LA CAPA CONSIDERANDO $\varepsilon-\mathrm{Fe}_{2} \mathrm{~N}_{1-\mathrm{y}} \mathrm{Y}$ $\gamma^{\prime}-\mathrm{Fe}_{4} \mathrm{~N}_{1-\mathrm{x}}$}

En el modelo de difusión se consideró que la fase $\gamma^{\prime}-\mathrm{Fe}_{4} \mathrm{~N}_{1-\mathrm{x}}$ es la primera en formarse y después sobre esta se forma la fase $\varepsilon-\mathrm{Fe}_{2} \mathrm{~N}_{1-y}$. Los tiempos de incubación de las fases son independientes $\mathrm{y}$ diferentes $t_{0}^{F e_{2} N_{1-y}}(T)>t_{0}^{F e_{4} N_{1-x}}(T)$. De tal manera que las condiciones iniciales $\mathrm{y}$ las de frontera para $C_{\mathrm{Fe}_{2} \mathrm{~N}_{1-y}}[x(t)]$ son: $t^{\prime}=0, \quad x>0$, donde: $C_{i}[x(t)]=C_{0}$, con: $i=\mathrm{Fe}_{4} \mathrm{~N}_{1-x}, \mathrm{Fe}_{2} \mathrm{~N}_{1-y}, C_{\mathrm{Fe}_{2} \mathrm{~N}_{1-y}}\left[x=u_{0} \approx 0\right]=C_{u p}^{\varepsilon}, C_{\mathrm{Fe}_{2} \mathrm{~N}_{1-y}}[x=u]=C_{\text {low }}^{\varepsilon}, C_{\mathrm{Fe}_{4} N_{1-x}}[x=u]=$ $C_{\text {int }}^{\gamma^{\prime}}$ y $C_{\mathrm{Fe}_{4} N_{1-x}}[x=v]=C_{\text {low }}^{\gamma^{\prime}}[8,9], u_{0}$ representa la película base de la etapa de nucleación $\approx 0$ en comparación a el espesor de $u$. Bajo las condiciones de frontera se obtiene el perfil de concentración lineal del nitrógeno en la fase $\mathrm{Fe}_{2} \mathrm{~N}_{1-\mathrm{y}}$ :

$$
C_{F_{2} N_{1-y}}[x(t)]=C_{u p}^{\varepsilon}+\frac{C_{l o w}^{\varepsilon}-C_{u p}^{\varepsilon}}{u} x
$$

Bajo las suposiciones antes mencionadas, se puede implementar un par de ecuaciones (3) y (7) basadas en la conservación de la materia en las interfases de crecimiento:

$$
C_{\text {low }}^{\varepsilon}+C_{\text {int }}^{\gamma^{\prime}}+\left(\frac{C_{u p}^{\varepsilon}-C_{l o w}^{\varepsilon}}{2}\right)
$$

El avance de la interfase $\mathrm{Fe}_{2} \mathrm{~N}_{1-y}$ y $\mathrm{Fe}_{4} \mathrm{~N}_{1-x}$ es: $\Delta C_{N}^{\varepsilon / \gamma^{\prime}}=J_{\varepsilon} d t-J_{\gamma^{\prime}} d t$ [9].Considerando una área unitaria plana en la interface $\mathrm{Fe}_{2} \mathrm{~N}_{1-\mathrm{y}} / \mathrm{Fe}_{4} \mathrm{~N}_{1-x}$ y $\mathrm{Fe}_{4} \mathrm{~N}_{1-\mathrm{x}} / \mathrm{Fe}$ y una distancia $d u$ en un tiempo $d t_{u}\left(t_{u}\right.$ representa el tiempo de formación de la fase), entonces se forma un volumen $(d u \cdot 1 \cdot 1)$. Lo anterior nos indica que el incremento de $d u$ del espesor de capa de $\mathrm{Fe}_{2} \mathrm{~N}_{1-y}$ en un tiempo $d t_{u}$ 
ocurre bajo el consumo simultáneo de la capa de espesor del $d u$ substrato. La acumulación de átomos de nitrógeno en el incremento $d u$ se puede expresar de la siguiente manera:

$$
\left(\frac{C_{l o w}^{\varepsilon}-2 C_{i n t}^{\gamma^{\prime}}+C_{u p}^{\varepsilon}}{2}\right)(1 \cdot 1 \cdot d u)=J_{\varepsilon}(x=u)\left(1 \cdot 1 \cdot d t_{u}\right)-J_{\gamma^{\prime}}(x=u+d u)\left(1 \cdot 1 \cdot d t_{u}\right)
$$

Considerado un perfil lineal de concentración de la fase $\gamma^{\prime}-\mathrm{Fe}_{4} \mathrm{~N}_{1-x}$, la pendiente del flujo se puede deducir con los términos $\boldsymbol{u}, \boldsymbol{v}$ y $\boldsymbol{l}$, (ver Figura 1), y se puede escribir de la siguiente forma:

$$
\frac{C_{l o w}^{\gamma^{\prime}}-C_{\text {int }}^{\gamma^{\prime}}}{l}=\frac{C_{\text {low }}^{\gamma^{\prime}}-C_{u p}^{\gamma^{\prime}}}{v}=\frac{C_{\text {int }}^{\gamma^{\prime}}-C_{u p}^{\gamma^{\prime}}}{u} .
$$

Sustituyendo la ecuación (9) en la ecuación (8):

$$
\left(\frac{C_{l o w}^{\varepsilon}-2 C_{i n t}^{\gamma^{\prime}}+C_{u p}^{\varepsilon}}{2}\right)\left(\frac{d u}{d t_{u}}\right)=\left[-D_{\varepsilon}\left(\frac{C_{l o w}^{\varepsilon}-C_{u p}^{\varepsilon}}{u}\right)\right]-\left[D_{\gamma^{\prime}}\left(\frac{C_{u p}^{\gamma^{\prime}}-C_{i n t}^{\gamma^{\prime}}}{u}\right)\right] .
$$

La ley de crecimiento parabólico $u=k_{\varepsilon} t_{u}^{m}$, se deriva y se sustituye en la ecuación (10), por lo tanto: $u=k_{\varepsilon}\left\{t^{\prime}-\left[t_{0}^{\varepsilon}(T)\right]\right\}^{1 / 2}$ donde $\left[t_{0}^{\varepsilon}(T)\right]^{1 / 2}$ representa el tiempo de incubación. Finalmente despejando se obtiene la relación del coeficiente de difusión de la fase $\varepsilon-\mathrm{Fe}_{2} \mathrm{~N}_{1-y}$ :

$$
D_{\varepsilon}=\frac{\left(C_{\text {low }}^{\varepsilon}-2 C_{\text {int }}^{\gamma^{\prime}}+C_{u p}^{\varepsilon}\right)\left(k_{\varepsilon}^{2}\right)+4 D_{\gamma^{\prime}}\left(C_{u p}^{\gamma^{\prime}}-C_{\text {int }}^{\gamma^{\prime}}\right)}{4\left(C_{u p}^{\varepsilon}-C_{l o w}^{\varepsilon}\right)} \cdot\left(m^{2} s^{-1}\right)
$$

\section{PROCEDIMIENTO EXPERIMENTAL}

\subsection{PROCESO DE NITRURACIÓN}

El material utilizado para ser nitrurado fue hierro Armco, con una composición química de $<200$ ppm de $\mathrm{C},<800$ ppm de $\mathrm{Mn},<200$ ppm de $\mathrm{P}$ y $<150$ ppm de S. La muestras fueron de forma cúbica con una dimensión de $8.5 \mathrm{~mm} \times 8.5 \mathrm{~mm} \times 5 \mathrm{~mm}$. Antes del proceso de nitruración las muestras fueron pulidas y se les realizó un decapado y pasivado con ácido sulfúrico, para retirar óxidos y otros contaminantes como grasa, aceites y pinturas. Posterior las muestras se limpiaron por ultrasonido en una solución de alcohol y agua des ionizada durante 5 min a temperatura ambiente, y se secaron. El contenedor es una caja cilíndrica cerrada (AISI 1018), al cual previamente se le realizó un tratamiento de nitruración para saturar sus paredes internas. Las muestras fueron embebidas dentro del contenedor con una mezcla de $\mathrm{CaCN}_{2}$. El tratamiento se realizó en un horno convencional con: cuatro valores de temperatura $(773,798,823$ y $848 \mathrm{~K}$ ) y cinco tiempos de tratamiento $(2,4,6,8$ y $12 \mathrm{~h})$ y tres relaciones activador/polvo rico en nitrógeno $(0.20,0.25 \mathrm{y}$ 0.35). Una vez terminado el tratamiento, el recipiente se retiró del horno y se dejó enfriar a temperatura ambiente.

\subsection{TECNICAS EXPERIMENTALES}


La muestras fueron cortadas transversalmente empleando un disco de diamante de la serie $15 \mathrm{HC}$, y se les realizó una preparación metalográfica. Para poder observar las secciones transversales de las capas de nitruro formadas se utilizó un microscopio electrónico de barrido (SEM) JEOL JSM 6300 LV. Para el estudio cinético, el espesor de la capa de nitruro se midió con la ayuda de software MSQ PLUS. Para asegurar la reproducibilidad de las capas medidas, cincuenta mediciones fueron tomadas de diferentes secciones de las muestras nitruradas para estimar el espesor de las capas $\gamma^{\prime}-\mathrm{Fe}_{4} \mathrm{~N}_{1-x}$ y $\varepsilon-\mathrm{Fe}_{2} \mathrm{~N}_{1-y}$; definidas como un valor medio de los dientes largo nitruro [10]. La presencia de los nitruros de hierro formado en la superficie de la muestra nitrurada se determinó por medio de equipos de Difracción de Rayos X (XRD) (D8-Focus) utilizando radiación CoK $\alpha$ en $\lambda=$ $1.05406 \mathrm{~nm}$. La distribución elemental dentro de la sección transversal de la capa de nitruro se determinó por espectroscopia de dispersión de electrones (EDS) equipo (JEOL JSM 6300 LV).

\section{RESULTADOS Y DISCUSIÓN}

\subsection{OBSERVACIONES EN SEM}

La Figura 2 muestra las secciones transversales de las capas de nitruro formados en la superficie del hierro Armco a 823 K, 6 y 12 h de tiempo de tratamiento y una relación de activador/polvo rico en nitrógeno 0.35. La microestructura resultante muestra la formación de la monocapa $\gamma^{\prime}-\mathrm{Fe}_{4} \mathrm{~N}_{1-\mathrm{x}}$ o la bicapa $\varepsilon-\mathrm{Fe}_{2} \mathrm{~N}_{1-y} / \gamma^{\prime}-\mathrm{Fe}_{4} \mathrm{~N}_{1-x}$, las cuales se observan densas, compactas y homogéneas, con una morfología semiplana. Además, se observa que, el crecimiento de las capas dependen de: la temperatura, el tiempo de tratamiento y la relación activador/polvo rico en nitrógeno.

"[Aquí va la Figura 2]"

Figura 2. Crecimiento de la monocapa $\gamma^{\prime}-\mathrm{Fe}_{4} \mathrm{~N}_{1-\mathrm{x}}$ o bicapa $\varepsilon-\mathrm{Fe}_{2} \mathrm{~N}_{1-\mathrm{y}} / \gamma^{\prime}-\mathrm{Fe}_{4} \mathrm{~N}_{1-\mathrm{x}}$ formadas en la superficie de hierro Armco, bajo las siguientes condiciones: temperatura $823 \mathrm{~K}$, relación activador/polvo rico en nitrógeno de 0.35 , tiempo de tratamiento: (a) $6 \mathrm{~h}, \mathrm{y}$ (b) $12 \mathrm{~h}^{[9]}$.

\subsection{ANÁLISIS EDS}

El análisis EDS obtenido por SEM se muestra en la Figura 3. Los resultados obtenidos señalan que en el caso del nitrógeno, se observa que va en aumento de $\gamma^{\prime}-\mathrm{Fe}_{4} \mathrm{~N}_{1-x}$ a $\varepsilon$ - $\mathrm{Fe}_{2} \mathrm{~N}_{1-\mathrm{y}}$. Asimismo, el carbono se presenta en mayor concentración en el sustrato, y disminuye en $\gamma^{\prime}-\mathrm{Fe}_{4} \mathrm{~N}_{1-\mathrm{x}} \mathrm{y}$ con menor disolución en la capa $\varepsilon-\mathrm{Fe}_{2} \mathrm{~N}_{1-y}$. Esto indica que el carbono tiende a ser desplazado por la capa nitrurada en dirección al substrato.

"[Aquí va la Figura 3]" 
Figura 3. EDS en tres zonas de la capa generada por nitruración en polvo sobre la superficie de un hierro Armco, bajo las siguientes condiciones: tiempo de tratamiento $6 \mathrm{~h}$, temperatura de $823 \mathrm{~K}$ y una relación activador/polvo rico en nitrógeno de 0.35. (a) zona de capa nitrurada $(\varepsilon)$, (b) zona de difusión $\left(\gamma^{\prime}\right)$, y (b) zona de difusión ${ }^{[9]}$.

\subsection{ANÁLISIS DE DIFRACCIÓN DE RAYOS X}

La Figura 4 muestra el XRD de la superficie de dos piezas nitruradas de hierro Armco, y se confirmó la formación de las fases $\varepsilon-\mathrm{Fe}_{2} \mathrm{~N}_{1-y} \mathrm{y} \gamma^{\prime}-\mathrm{Fe}_{4} \mathrm{~N}_{1-\mathrm{x}}$. En estas difracciones los picos son diferentes de intensidad y dependen de las orientaciones cristalográficas de los cristales de $\varepsilon-\mathrm{Fe}_{2} \mathrm{~N}_{1-\mathrm{y}} \mathrm{y} \gamma^{\prime}-\mathrm{Fe}_{4} \mathrm{~N}_{1-}$ $x$. En un punto de vista cinético los cristales comienzan su nucleación en la superficie del substrato, cuando la matriz alcanza el nivel de saturación por átomos de nitrógeno, y posterior la coalición de estos núcleos se convierte en una capa $\gamma^{\prime}-\mathrm{Fe}_{4} \mathrm{~N}_{1-x}$, posterior cuando la aumenta la saturación sobre la capa $\gamma^{\prime}-\mathrm{Fe}_{4} \mathrm{~N}_{1-x}$ se inicia la formación de la capa $\varepsilon-\mathrm{Fe}_{2} \mathrm{~N}_{1-y}$ bajo el mismo mecanismo anterior.

"[Aquí va la Figura 4]"

Figura 4. Patrón de difracción de rayos $X(X R D)$, originado durante la detección de las fases $\gamma^{\prime}-\mathrm{Fe}_{4} \mathrm{~N}_{1-\mathrm{x}} \mathrm{y} \varepsilon-\mathrm{Fe}_{2} \mathrm{~N}_{1-y}$, generadas en la superficie de hierro Armco bajo las siguientes condiciones: (a) tiempo de tratamiento $4 \mathrm{~h}$, temperatura de $823 \mathrm{~K}$ y una relación activador/polvo rico en nitrógeno de 0.25 ; (b) tiempo de tratamiento $12 \mathrm{~h}$, temperatura de 823 $\mathrm{K}$ y una relación activador/polvo rico en nitrógeno de $0.25^{[9]}$.

\subsection{CINÉTICA DE CRECIMIENTO DE LAS FASES $\varepsilon_{-} \mathrm{Fe}_{2} \mathrm{~N}_{1-\mathrm{y}} \mathrm{Y} \gamma^{\prime}-\mathrm{Fe}_{4} \mathrm{~N}_{1-\mathrm{x}}$}

Con los resultados obtenidos de la medición de espesores de capas generadas se graficó $v^{2}$ vs $t^{\prime}$ y $u^{2}$ vs $t^{\prime}$, como se puede observar en la Figura 5. Las pendientes de las líneas rectas representan las constantes de crecimiento parabólico $k_{\text {total }}^{2}$ y $k_{\varepsilon}^{2}$ respectivamente, y su intersección con el eje de las abscisas indica el tiempo de incubación de la capa.

"[Aquí va la Figura 5]"

Figura 5. Evolución de los espesores de capa generada en hierro Armco con una relación activador/polvo rico en nitrógeno 0.25 , (a) capa $\varepsilon-\mathrm{Fe}_{2} \mathrm{~N}_{1-y}+\gamma^{\prime}-\mathrm{Fe}_{4} \mathrm{~N}_{1-x}$ (b) capa $\varepsilon-\mathrm{Fe}_{2} \mathrm{~N}_{1-y}{ }^{[9]}$.

Asumiendo la relación de Arrhenius, y utilizando las constantes de crecimiento parabólico obtenidas para las fases $\gamma^{\prime}-\mathrm{Fe}_{4} \mathrm{~N}_{1-\mathrm{x}}$ y $\varepsilon-\mathrm{Fe}_{2} \mathrm{~N}_{1-y}$, se obtienen las energías de activación $Q_{\gamma^{\prime}}$ y $Q_{\varepsilon}$, en la Figura 6 se indica la energía necesaria para estimular la difusión del nitrógeno. 
Figura 6. Gráficas de Arrhenius para la nitruración en polvo de un hierro Armco, con una relación activador/polvo rico en nitrógeno 0.25 , (a) fase $\gamma^{\prime}-\mathrm{Fe}_{4} \mathrm{~N}_{1-x}$ (b) fase $\varepsilon-\mathrm{Fe}_{2} \mathrm{~N}_{1-y}{ }^{[9]}$.

Tabla 1 Energía de activación y factor pre-exponencial para formar las fases $\varepsilon-\mathrm{Fe}_{2} \mathrm{~N}_{1-y} \mathrm{y} \gamma^{\prime}$ - $\mathrm{Fe}_{4} \mathrm{~N}_{1-\mathrm{x}}$ por difusión de nitrógeno en hierro puro.

\begin{tabular}{|c|c|c|c|c|}
\hline $\begin{array}{c}\text { Relación } \\
\text { activador/Polvo rico en nitrógeno }\end{array}$ & $\begin{array}{c}D_{0}^{\gamma^{\prime}} \\
\left(\mathbf{m}^{2} \mathbf{s}^{-1}\right)\end{array}$ & $\begin{array}{c}Q_{\gamma^{\prime}} \\
\left(\mathrm{Jmol}^{-1}\right)\end{array}$ & $\begin{array}{c}D_{0}^{\varepsilon} \\
\left(\mathbf{m}^{2} \mathbf{s}^{-1}\right)\end{array}$ & $\begin{array}{c}Q_{\varepsilon} \\
\left(\mathrm{Jmol}^{-1}\right)\end{array}$ \\
\hline 0.20 & $7.82 \times 10^{-9}$ & 81211.98 & $1.35 \times 10^{-9}$ & 89599.98 \\
\hline 0.25 & $9.84 \times 10^{-9}$ & 79549.18 & $2.47 \times 10^{-9}$ & 89624.92 \\
\hline 0.35 & $1.64 \times 10^{-8}$ & 79535.88 & $4.13 \times 10^{-9}$ & 89325.62 \\
\hline
\end{tabular}

Como se observa en la Tabla 1, la energía de activación no representa cambios en función de las relaciones activador /polvo, siendo una propiedad intrínseca de las fases de nitruros de hierro. Por lo tanto, se puede tomar un promedio para obtener los valores de $Q_{\gamma^{\prime}}$ y $Q_{\varepsilon}$ en el conjunto experimental de la nitruración en polvo, estos valores coinciden con la literatura [11-13]. Por otro lado se observa que el coeficiente de difusión está influenciado por el porcentaje de activador utilizado, ya que conforme aumenta la relación activador/polvo rico en nitrógeno, teniendo en cuenta todo lo anterior se obtienen los coeficientes de difusión como se observa en la Figura 7.

"[Aquí va la Figura 7]"

Figura 7. Influencia de la relación activador/polvo rico en nitrógeno, en el coeficiente de difusión para la nitruración por polvo en la superficie de hierro Armco, (a) fase $\gamma^{\prime}-\mathrm{Fe}_{4} \mathrm{~N}_{1-\mathrm{x}}$ (b) fase $\varepsilon-\mathrm{Fe}_{2} \mathrm{~N}_{1-\mathrm{y}}{ }^{[9]}$.

Utilizando la ecuacion de relación activador/polvo rico en nitrogeno, obtenemos las relaciones tipo Arrhenius para los coeficientes de difusión del nitrogeno en las fases $\gamma^{\prime}-\mathrm{Fe}_{4} \mathrm{~N}_{1-\mathrm{x}}$ y $\varepsilon-\mathrm{Fe}_{2} \mathrm{~N}_{1-\mathrm{y}}$ :

$$
\begin{aligned}
& D_{\gamma^{\prime}}=\left(6.35 \times 10^{-10} \mu-4.56 \times 10^{-9}\right) \exp \left(-\frac{80.1 \pm 0.9 \mathrm{kJmol}^{-1}}{R T}\right), \quad\left(\mathrm{m}^{2} \mathrm{~s}^{-1}\right) \\
& D_{\varepsilon}=\left(1.89 \times 10^{-10} \mu-2.28 \times 10^{-9}\right) \exp \left(-\frac{89.5 \pm 0.2 \mathrm{kJmol}^{-1}}{R T}\right) . \quad\left(\mathrm{m}^{2} \mathrm{~s}^{-1}\right)
\end{aligned}
$$

Finalmente utilizando las ecuaciones de Arrhenius (12) y (13) se pueden reecribir las ecuaciones de crecimiento parabólico para los espesores de las capas $\gamma^{\prime}-\mathrm{Fe}_{4} \mathrm{~N}_{1-\mathrm{x}}, \mathrm{y} \varepsilon-\mathrm{Fe}_{2} \mathrm{~N}_{1-\mathrm{y}}$.

$$
v=\left(\frac{D_{\gamma^{\prime}} 4 t^{\prime}\left(C_{u p}^{\gamma^{\prime}}-C_{\text {low }}^{\gamma^{\prime}}\right)}{\left(C_{\text {low }}^{\gamma^{\prime}}-2 C_{0}+C_{\text {up }}^{\gamma^{\prime}}\right)}\right)^{1 / 2}\left(1-\frac{t_{0}^{\gamma^{\prime}}(T)}{t^{\prime}}\right)^{1 / 2}, \quad(m)
$$




$$
u=\left(\frac{D_{\varepsilon} 4 t^{\prime}\left(C_{u p}^{\gamma^{\prime}}-C_{\text {low }}^{\gamma^{\prime}}\right)-4 D_{\gamma^{\prime}}\left(C_{u p}^{\gamma^{\prime}}-C_{\text {int }}^{\gamma^{\prime}}\right)}{\left(C_{\text {low }}^{\varepsilon}-2 C_{\text {int }}^{\gamma^{\prime}}+C_{u p}^{\varepsilon}\right)}\right)^{1 / 2}\left(1-\frac{t_{0}^{\varepsilon}(T)}{t^{\prime}}\right)^{1 / 2} \cdot \quad(m)
$$

En la Tabla 2 se presenta la comparación de los valores calculados con las ecuaciones (14) y (15) y los valores obtenidos experimentalmente.

Tabla 2 Comparación de los valores experimentales, y los valores calculados de los espesores de capas nitruradas formadas superficialmente bajo la siguientes condiciones: (a) temperatura $798 \mathrm{~K}$, tiempo de tratamiento 9 h, relación activador/polvo rico en nitrógeno 0.25; (b) temperatura 823 K, tiempo de tratamiento 9 h, relación activador/polvo rico en nitrógeno 0.35 .

\begin{tabular}{|c|c|c|c|c|}
\hline Fases & $\begin{array}{l}\text { Relación activador/polvo } \\
\text { rico en nitrógeno }\end{array}$ & $\begin{array}{c}\text { Espesor de capa estimado a } \\
\text { partir de las ecuaciones }(14) \text { y } \\
(15)(\mu \mathrm{m})\end{array}$ & $\begin{array}{c}\text { Espesor de capa } \\
\text { experimental }(\mu \mathrm{m})\end{array}$ & $\begin{array}{c}\text { Error } \\
(\%)\end{array}$ \\
\hline $\begin{array}{c}\gamma^{\prime}-\mathrm{Fe}_{4} \mathrm{~N}_{1-\mathrm{x}} \\
(v)\end{array}$ & \multirow[t]{2}{*}{0.25} & 8.7 & $8.4 \pm 1.2$ & 3.5 \\
\hline $\begin{array}{c}\varepsilon-\mathrm{Fe}_{2} \mathrm{~N}_{1-\mathrm{y}} \\
(u)\end{array}$ & & 4.6 & $5.0 \pm 0.9$ & 8.0 \\
\hline $\begin{array}{c}\gamma^{\prime}-\mathrm{Fe}_{4} \mathrm{~N}_{1-\mathrm{x}} \\
(v)\end{array}$ & \multirow[t]{2}{*}{0.35} & 14.9 & $15.2 \pm 1.2$ & 1.9 \\
\hline $\begin{array}{c}\varepsilon-\mathrm{Fe}_{2} \mathrm{~N}_{1-\mathrm{y}} \\
(u)\end{array}$ & & 9.9 & $9.6 \pm 0.7$ & 3.1 \\
\hline
\end{tabular}

\section{CONCLUSIONES}

En el presente trabajo se estimó la cinética de crecimiento de las capas $\gamma^{\prime}-\mathrm{Fe}_{4} \mathrm{~N}_{1-\mathrm{x}}$ y $\varepsilon$ - $\mathrm{Fe}_{2} \mathrm{~N}_{1-\mathrm{y}}$ formadas en la superficie de un hierro Armco, generadas por el proceso de nitruración en polvo. El cual fue evaluado usando el modelo de cinética de crecimiento propuesto, donde se utilizaron temperaturas en el rango de 798-848 K, tiempos de tratamiento 2-12 h, y relación activador/polvo rico en nitrógeno 0.20-0.35. El modelo fue basado en las ecuaciones de balance de masa $\left(\mathrm{Fe}_{2} \mathrm{~N}_{1}\right.$ y $/ \mathrm{Fe}_{4} \mathrm{~N}_{1-x}$ y $\mathrm{Fe}_{4} \mathrm{~N}_{1-x} / \mathrm{Fe}$ ) en la interfaces, considerando tiempos de incubación. Le energía de activación fue estimada en $Q_{\gamma^{\prime}}=80.1 \mathrm{KJmol}^{-1}$ y $Q_{\varepsilon}=89.5 \mathrm{KJmol}^{-1}$ por el proceso de nitruración en polvo sobre la superficie de hierro Armco dentro de un ranto de temperaturas de 798-848 K. El presente modelo fue validado por comparación con valores de espesores de capas nitruradas generadas de forma experimental utilizando un tiempo de tratamiento de $9 \mathrm{~h}$, dos temperaturas 798 y $823 \mathrm{~K}$, así como dos relaciones activador/ polvo rico en nitrógeno 0.25 y 0.35 respectivamente. 
Los autores aprecian la ayuda de la Maestra Alma Corral Lopez y. al Instituto Tecnológico de Estudios Superiores de Monterrey, Campus Santa Fe por el apoyo brindado a través de sus laboratorios especializados.

\section{Bibliografía}

${ }^{[1]}$ LÓPEZ, L. D., OROZCO, P. (1993). Estudio de Propiedades Físicas Superficiales de Acero 4140 nitrogenado con plasma. Revista Colombiana de Física, (25), 31-40.

${ }^{[2]}$ MEDINA-FLORES, A., ARGANIS, C., SANTIAGO, P., OSEGUERA, J. (2004). Electrochemicals corrosion tests of an AISISAE 4140 steel nitride by post-discharge microwave plasma. Surface \& Coatings Technology, (188-189), 140-145.

${ }^{[3]}$ MEDINA-FLORES, A., OSEGUERA, J., SANTIAGO, P., ASCENSIO, J.A. (2004). Structural analysis of AISI-SAE 4140 steel nitrided by post-discharge microwave. Surface \& Coatings Technology, (188-189), 7-12.

${ }^{[4]}$ MOO-CHIN, WANG., CHIEN, C. Y., NAN-CHUNG, W. (2003). Grain growth and electric properties of liquid phase sintered AIN ceramics with CaCN2 additives. Materials Science and Engineering, (343), 97-106.

${ }^{[5]}$ Calcium cyanamide and aminoguanidine from urea and calcium oxide, (2010). The art and science of amateur $\begin{array}{llllll}\text { experimentalism, } & \text { [viewed } & 7 & \text { August } & \text { 2015] }\end{array}$ http://www.sciencemadness.org/talk/viewthread.php?tid=14267

${ }^{[6]}$ FRAGUELA, A., CASTILLO, F., OSEGUERA, J. (2010). Formas de representación del transporte de nitrógeno atómico en el sistema Fe-N: Estudio del estado estacionario en un proceso de nitruración post-descarga. Revista Mexicana de Física, 56(5), 363-377.

${ }^{[7]}$ ROY, U. (1968). Phase boundary motion and polyphase diffusion in binary metal-interstitial systems. Acta Metallurgica, (16), 243-253.

${ }^{[8]}$ CAVAliereA, P., ZAVARISEA, G., PERILLOB, M. (2009). Modeling of the carburizing and nitriding processes. Computational Materials Science, 46(1), 26-35.

${ }^{[9]}$ ELIAS, Milton. (2013) Caracterización mecánica y cinética de crecimiento de fases nitruradas obtenidas por el proceso de nitruración en polvo. Tesis Doctorado. IPN.

${ }^{[10]}$ ORTIZ-DOMÍNGUEZ, M., FLORES-RENTERIA, M. KEDDAM, M., ELIAS-ESPINOSA, M., DAMIÁN-MEJÍA, O., ALDANAGONZÁLEZ, J., ZUNO-SILVA, J., MEDINA-MORENO, S., GONZÁLEZ-REYES, J. (2014). Simulation of growth kinetics of $\mathrm{Fe}_{2} B$ layers formed on gray cast iron during the powder-pack boriding. Materiali in tehnologije / Materials and technology, 48(6), 905-916.

${ }^{[11]}$ SOMERS, M., MITTEMEIJER, E. (1995) Layer-growth kinetics on gaseous nitriding of pure iron: Evaluation of diffusion coefficients for nitrogen in iron nitrides. Metallurgical and Materials Transactions, 26(1), 57-74. 
${ }^{[12]}$ BELMONTE, T., GOUNÉ, M., MICHEL, H. (2001). Numerical modeling of interstitial diffusion in binary systems. Application to iron nitriding. Materials Science and Engineering, (302), 246-257.

${ }^{[13]} \mathrm{Du}, \mathrm{H} .$, Agren, J. (1995). Gaseous nitriding iron: evaluation of diffusion data of $\mathrm{N}$ in $\gamma^{\prime}$ and $\varepsilon$ phases. Zeitschrift für Metallkunde, 86(8), 522-529. 